\title{
Remarks on the Surface Area and Equality Conditions in Regular Forms. Part I: Triangular Prisms
}

\author{
Ahmed A. Elkhateeb
}

Published online: 6 March 2014

(C) Kim Williams Books, Turin 2014

\begin{abstract}
This work presents four mathematical remarks concluded from the mathematical analysis for the interrelationships between the dependent and independent variables that control the measures: perimeter, floor area, walls surface area and total surface area in the regular forms that have a given volume. Such forms include prismatic and pyramidal forms. The work consists of four parts, of which this first part presents the remarks of the isosceles triangular right prism. The first remark examines the effect of $\theta$, the angle of the triangular base, on the total surface area. The second remark calculates the minimum total surface area in two cases, depending on whether angle $\theta$ is constant or variable. The third remark calculates the walls ratio and the critical walls ratio. The last remark studies the required conditions for the numerical equality in two cases, where the perimeter is equal to the area, and where the total surface area is equal to the volume.
\end{abstract}

Keywords Trigonometry · Algebra $\cdot$ Differential equations · Volume · Area - Total surface area $\cdot$ Perimeter $\cdot$ Regular polygons $\cdot$ Right triangular prisms $\cdot$ Minimum total surface area $\cdot$ Walls ratio $\cdot$ Numerical equality

\section{Introduction}

Form is the visual appearance of a three-dimensional object, and is often the main target in the architectural design. Form and space create the ambient world in which we live and experience our environment. According to Francis Ching, the form is established “... by the shapes and interrelationships of the planes that describe the boundaries of the volume" (Ching 2007, p. 28).

\footnotetext{
A. A. Elkhateeb $(\bowtie)$

Department of Architecture, Faculty of Environmental Designs, King Abdulaziz University, Jeddah, Saudi Arabia

e-mail: aelkhateeb@kau.edu.sa
} 
Mathematically, the perimeter (Per), floor area (Ar), total surface area (S) and volume $(\mathrm{V})$ are four basic measures that can describe numerically any form. For our purposes here, a fifth value is also significant, $\theta$, the angle of a triangular base. The interest in area and volume calculations has been started very early. It may go back to the period of the ancient Greeks (ca. eighth-sixth century BC) who calculated formulas to measure areas of simple geometrical shapes. Further, the ancient Greeks measured volumes according to their dry or liquid conditions suited, respectively to measuring grain and wine. In his "Elements", Euclid presented many axioms and postulates related to the area of simple geometrical shapes. Nowadays, the methods and formulas to calculate Per, Ar, $\mathrm{S}$ and $\mathrm{V}$ for the common forms are almost available in every mathematical textbook [see, for example, (Ferguson and Piggott 1923; Bird 2003; Gieck and Gieck 2006)].

In modern advanced building analysis and design (such as room acoustics, artificial or day lighting and environmental control), areas and volumes are crucial measures. For example, in room acoustics, the ratio between Per and Ar determines the shape factor $S h_{f}$. Also, the ratio between $\mathrm{V}$ and $\mathrm{S}$ of a room determines its mean free path $l$ and reverberation time T (Sabine 1993), both are basic measures in room acoustics (Elkhateeb 2012). In artificial lighting, room area is a main measure in the calculation of room cavity ratios that is a basic factor in lighting design (Grondzik et al. 2006). In environmental control, the areas of walls and/or roofs that face directly the sun control the amount of heat that transfers to the interior of a room according to the thermal connectivity and resistivity of their materials (Konya 2011). Thus, it is important for architects and practitioners in this field to be aware about the mathematical characteristics of these two measures (area and volume) and how they affect each other.

The regular forms that will be addressed in this work are divided into two main groups:

- The first includes all of the right prisms that have regular bases according to the definition of the term "regular" as used through this work (see Sect. "Definition of "Regular Forms" in this Work"). The plan is to cover the entire range of the regular basic shapes (from the triangle, to the circle). This group will be addressed in the first three parts of the work:

- Part I: triangular prisms (this present paper);

- Part II: quadratic prisms (including both: rectangle and trapezoid);

- Part III: multi-sides prisms (from pentagon to circle).

- The second includes all of the right regular pyramids either complete or incomplete (frustum of right pyramid). The base(s) has to be also a regular shape. These will be discussed in Part IV.

\section{Definition of "Regular Forms" in this Work}

In case of right prisms, the term "regular", as used in this part and in the subsequent parts of the work, means that the bases of the prism have at least one axis of symmetry. Thus, in right triangular prisms, for example, only isosceles or 
equilateral triangles will be considered. In quadratic shapes only rectangular or symmetrical (isosceles) trapezoidal shapes will be considered. In the third dimension, the room is a right prism (i.e., all the sides of the prism are rectangles).

In the case of right pyramids, the term "regular" means that the base is a regular polygon (i.e., a multi-sides shape, from the equilateral triangle to the circle). In the third dimension, the apex of this pyramid is aligned directly above the center of its regular base.

The regular forms (or rooms, both terms could be used through the work) as described above have been chosen as the subject of this work because they are the most common in architectural applications. In addition, they can be grouped, organized and mathematically analyzed utilizing a consistent methodology. Although irregular rooms can be also studied using the same methodology, they have to be investigated separately according to the assumptions of each case but not as a group as the regular ones.

\section{Problem Definition}

Basic mathematical formulas to calculate the different measures (mainly, Per, Ar, S and $\mathrm{V}$ ) of any regular form were established many years ago and are well-known. Nevertheless, to my knowledge, there is no advanced study that analyzes the interrelationships between these variables (dependent and independent). Consequently, a discussion of the way they affect each other is still lacking.

\section{Objectives}

The main aim of this work is to highlight specific cases that have a specific behavior in the advanced building analysis. For example, in a right prismatic room with isosceles triangular bases and a given volume (see "The Mathematical Relationships of Regular Triangular Prisms"), the work seeks a better understanding of the way in which the variables ( $\theta$, Per, Ar, S and V) affect each other. In particular, the work tries to draw clear conclusions about:

- How the angle $\theta$ (or $\theta$ and $\beta$ ) affects $S$;

- When $\mathrm{S}$ becomes minimum $\left(\mathrm{S}_{\mathrm{Min}}\right)$;

- The ratio between walls surface area $S_{\mathrm{W}}$ and $\mathrm{S}\left(\mathrm{S}_{\mathrm{W}} / \mathrm{S}=\mathrm{R}_{\mathrm{W}}\right)$;

- When Ar numerically equals Per;

- When $\mathrm{S}$ is numerically equal to $\mathrm{V}$.

\section{Methodology}

For the purpose of this work, and in the beginning, the rules of analytical geometry and trigonometry were utilized to derive a set of mathematical functions that relate the dependent variables Per, $\mathrm{H}_{\mathrm{R}}$ (height of the room; see Fig. 2) and $\mathrm{S}$ to the independent variables $\theta$, Ar and $\mathrm{V}$. In a room that has a given volume $\mathrm{V}$, there are 


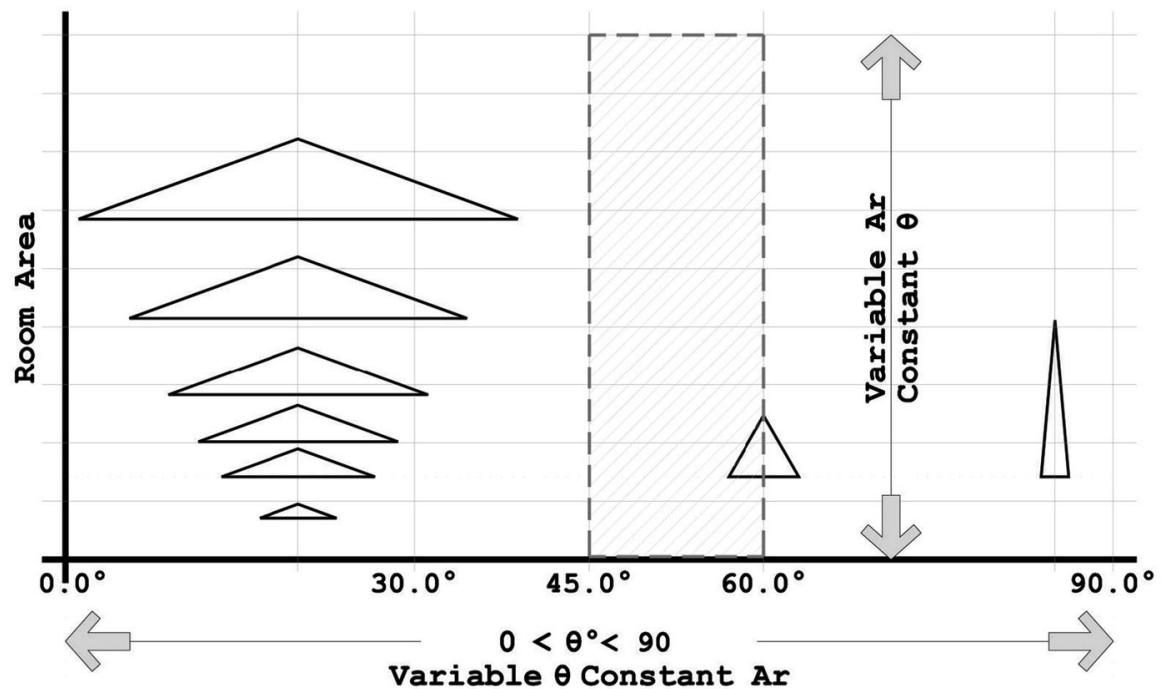

Fig. 1 The two tactics considered in this work, an example from the triangular prisms

different tactics to achieve this V. For the purpose of this work, only two tactics will be considered (see Fig. 1):

- Case of constant $\theta$ with variable $\mathrm{Ar}$, and $\mathrm{H}_{\mathrm{R}}$;

- Case of variable $\theta$ with constant $\mathrm{Ar}$ and $\mathrm{H}_{\mathrm{R}}$.

For both cases, the derived functions were used to examine the effect of $\theta$ (or $\theta$ and $\beta$ ) on $S$ and to calculate $S_{\text {Min }}$ for the room under discussion. Finally, the conditions for the equality were calculated utilizing the rules of algebra and trigonometry based on the derived functions.

\section{The Mathematical Relationships of Regular Triangular Prisms}

In an isosceles triangular right prism and for the purpose of this work, it is assumed that the angle $\theta$, Ar and $\mathrm{V}$ are the independent variables whereas Per and $\mathrm{S}$ are the dependent ones. This section derives the main mathematical functions among these two groups of variables. During the analysis, the effect of only one independent variable on the other variables will be considered. Fig. 2 represents the different terms that will be used in this analysis, namely: $\theta$ (the base angle of the triangle), a (the side of the triangle), $b$ (the base of the triangle), $h$ (the height or altitude of the triangle) and $\mathrm{H}_{\mathrm{R}}$ (the height of the room).

An isosceles triangle can be completely identified knowing both Ar and $\theta$. From the first principles, it can be concluded that:

$$
h=\sqrt{\frac{A r \sin \theta}{\cos \theta}}
$$



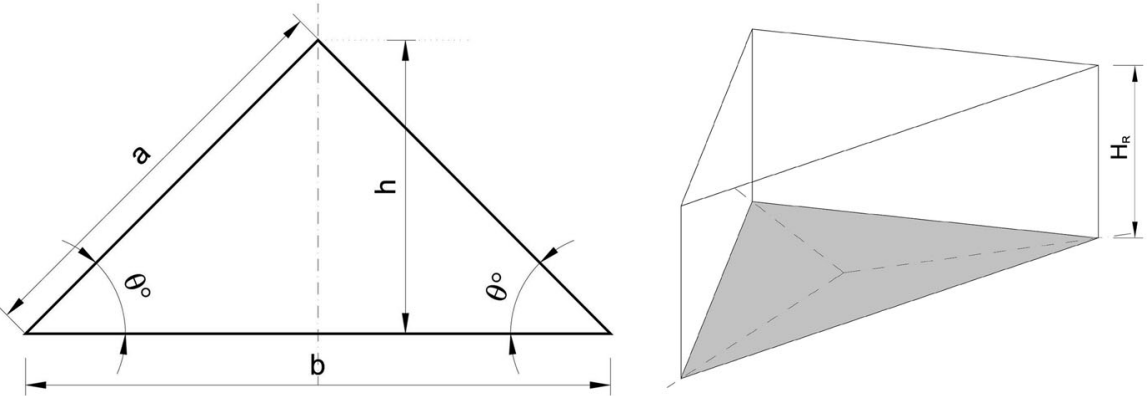

Fig. 2 Isosceles triangular rooms, the different variables. a left, Room plan, b right, Room 3-D and

$$
b=\frac{2 h}{\tan \theta} \text {. }
$$

Thus

$$
\operatorname{Per}=2 h\left(\frac{1+\cos \theta}{\sin \theta}\right)
$$

and

$$
A r=\frac{h^{2}}{\tan \theta} .
$$

In the third dimension, an isosceles triangular shape can be extruded to form a right prism. In this case, its volume $\mathrm{V}$ can be calculated from:

$$
V=\frac{h^{2} H_{R}}{\tan \theta}
$$

Consequently

$$
H_{R}=\frac{V \tan \theta}{h^{2}}
$$

and the total surface area $\mathrm{S}$ of a right prism with isosceles triangular bases can be calculated as:

$$
S=2 A r+\left(\operatorname{Per} \times H_{R}\right) .
$$

Given the values of Per (Eq. 3), Ar (Eq. 4), and $\mathrm{H}_{\mathrm{R}}$ (Eq. 6) as a function of $\theta$, Eq. 7 can be rewritten as either:

$$
S=\frac{2 h^{2}}{\tan \theta}+2 h H_{R}\left(\frac{1+\cos \theta}{\sin \theta}\right)
$$

or:

$$
S=\frac{2 h^{2}}{\tan \theta}+2 \frac{V \tan \theta}{h}\left(\frac{1+\cos \theta}{\sin \theta}\right) .
$$




\section{Remark 1: Effect of $\theta$ on $S$}

Figure. 3 is a graphical representation of Eq. 8. As can be concluded from this figure, the behavior of the function changes dramatically from one zone to another based on the value of $\theta$. The function is semi-symmetrical and reaches its minimum at $\theta=60^{\circ}$. This angle, $\theta=60^{\circ}$, splits the function into two main zones:

- Zone 1: This zone encloses between $0^{\circ}<\theta \leq 60^{\circ}$, in this zone $\mathrm{S}$ is a decreasing function of $\theta$. This zone can be also divided into two sub-zones:

- Zone of rapid decay (a) $\left(0^{\circ}<\theta \leq 15^{\circ}\right)$ : where $S$ loses about $60 \%$ of its maximum value.

- Zone of slow decay (b) $\left(15^{\circ} \leq \theta \leq 60^{\circ}\right)$ : $\theta$ increases rapidly in comparison with the reduction in $\mathrm{S}$ (in this zone, $\mathrm{S}$ loses about $22 \%$ of its value at $\left.\theta=15^{\mathrm{o}}\right)$.

- Zone 2: in this zone $\mathrm{S}$ is an increasing function of $\theta$. This zone (between $60^{\circ} \leq \theta<90^{\circ}$ ) can be also divided into two additional sub-zones (c) (up to $\theta \leq 85^{\circ}$ ), and (d). Both zones are almost identical to the sub-zones (b) and (a), respectively.

It can also be concluded from Fig. 3 that the variation in $\mathrm{S}$ corresponding to $\theta$ in the range between $45^{\circ}$ and $60^{\circ}$ is limited and can be ignored (Elkhateeb 2012). Nevertheless, beyond this range (whether $\theta \geq 60^{\circ}$ or $\theta \leq 45^{\circ}$ ) this variation is obvious and must be considered upon deciding the dimensions and setups of a room. One can argue that values of $\theta$ outside this range $\left(45^{\circ}-60^{\circ}\right)$ are not common in

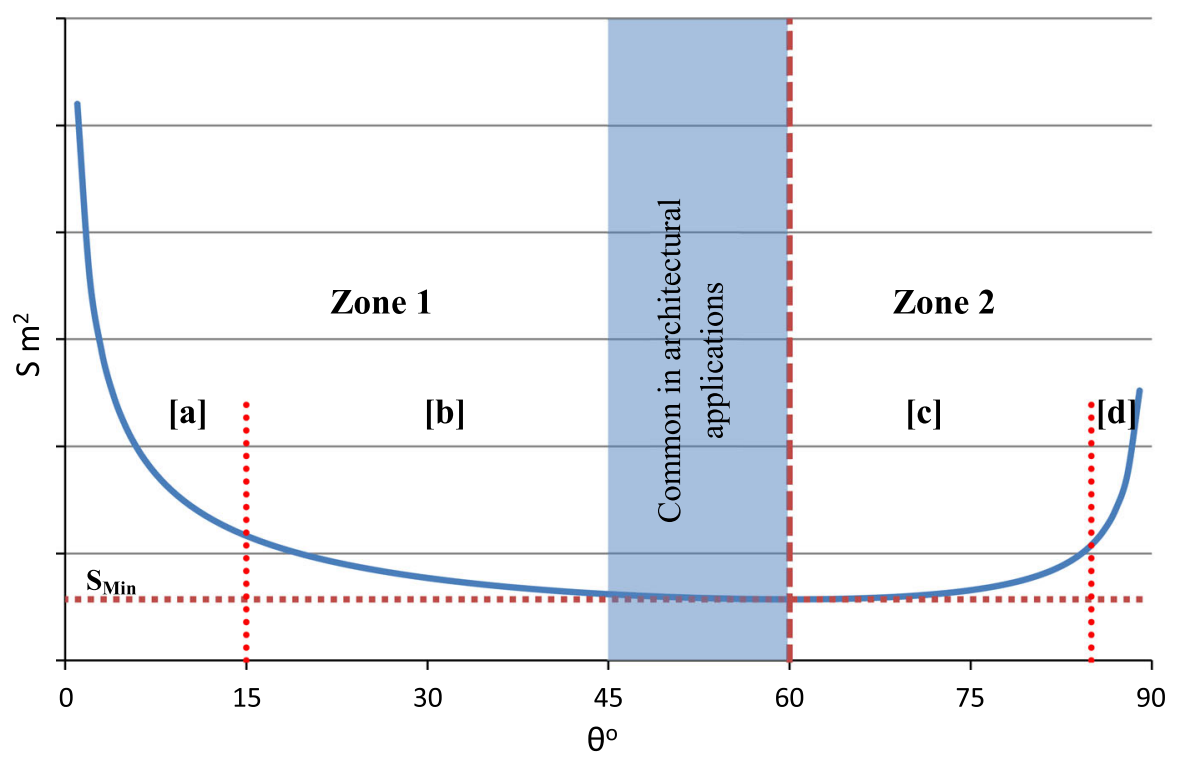

Fig. 3 The relationship between $\theta$ and $\mathrm{S}$, graphical representation of Eq. 8 
architectural applications. However, we cannot rely on that, as everything is possible in architecture. More discussion about the relationship between $\theta$ and $S$ is presented in "Case II, Variable $\theta$, Constant Ar and $\mathrm{H}_{\mathrm{R}}$ ".

\section{Remark 2: the Minimum Total Surface Area, $S_{M i n}$}

In a prismatic room that has isosceles triangular bases and a given $\mathrm{V}, \mathrm{S}_{\text {Min }}$ depends on the case of the angle $\theta$, which could be either:

- Constant, in this case both $\mathrm{Ar}$ and $\mathrm{H}_{\mathrm{R}}$ will be the variables, or

- Variable, in this case both $\mathrm{Ar}$ and $\mathrm{H}_{\mathrm{R}}$ will be constants.

Thus, Remark 2 will be divided into two sub-remarks in order to discuss both cases.

Case I, Constant $\theta$, Variable $\mathrm{Ar}$ and $\mathrm{H}_{\mathrm{R}}$

In this case, among the different isosceles triangular rooms that have the same $\theta$ and $\mathrm{V}, \mathrm{S}_{\mathrm{Min}}$ occurs when the first derivative of Eq. 9 equals zero, i.e.:

$$
\frac{d S}{d h}=\frac{4 h}{\tan \theta}-2 \frac{V \tan \theta}{h^{2}}\left(\frac{1+\cos \theta}{\sin \theta}\right)=0 .
$$

This leads to:

$$
h^{3}=\frac{V}{2} \tan ^{2} \theta\left(\frac{1+\cos \theta}{\sin \theta}\right) .
$$

From Eq. 5, the last formula (Eq. 11) can be rewritten as:

$$
h=\frac{H_{R} \tan \theta}{2}\left(\frac{1+\cos \theta}{\sin \theta}\right) \text {. }
$$

Thus,

$$
\frac{H_{R}}{h}=\frac{2 \sin \theta}{\tan \theta+\sin \theta} .
$$

Equations 12 and 13 indicate the conditions under which $\mathrm{S}$ will assume its minimum value. The ratio $H_{R} / h$ will be called $\omega$. When $\omega$ results $S_{\text {Min }}$, it will be called the critical ratio $\omega_{o}$. Thus, Eq. 13 can be rewritten as

$$
\omega_{o}=\frac{2 \sin \theta}{\tan \theta+\sin \theta} \text {. }
$$

Applying the rules of trigonometry, this last formula can be simplified to:

$$
\omega_{o}=\frac{2}{\sec \theta+1} \text {. }
$$

As can be concluded from Eq. 15, $\omega_{o}$ depends entirely on $\theta$ : for every $\theta$ there is a specific $\omega_{o}$ that produces $S_{\text {Min }}$. A room that has such dimensions possesses the minimum total surface area among other rooms that have the same $\mathrm{V}$ and $\theta$. The 


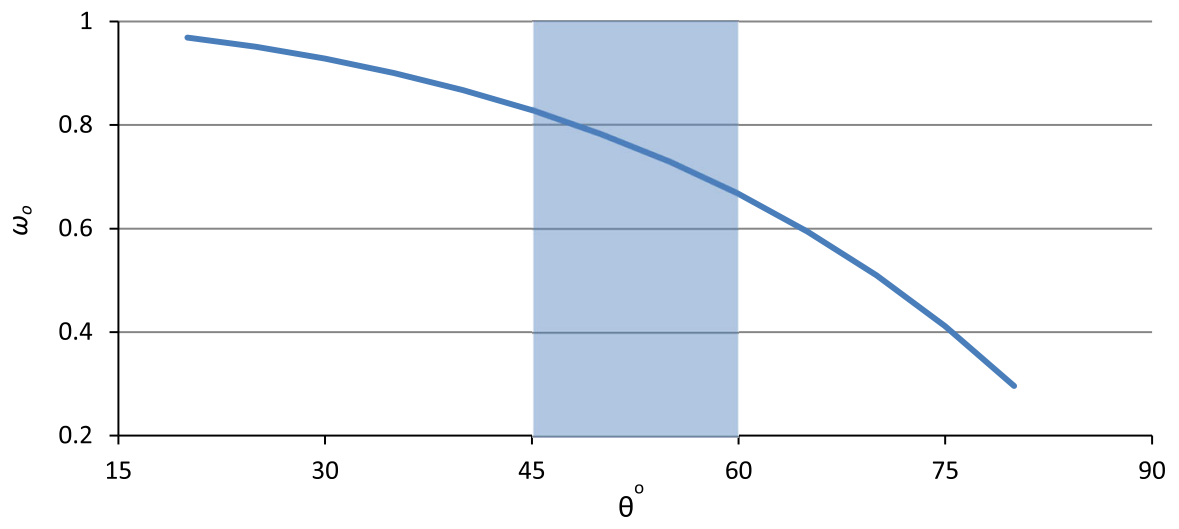

Fig. 4 Values of $\omega_{o}$ in the range $20^{\circ} \leq \theta \leq 80^{\circ}$ according to Eq. 15

values of $\omega_{o}$ were calculated for the range $20^{\circ} \leq \theta \leq 80^{\circ}$, and results are presented in Fig. 4. It is clear from this figure that $\omega_{o}$ is a decreasing function of $\theta$. The shaded zone in this figure indicates the common zone in architectural applications.

To determine room dimensions that fulfill $\mathrm{S}_{\mathrm{Min}}$, the following methodology can be applied:

- Determine both $\theta$ and $\mathrm{V}$ of the room;

- Calculate $\omega_{o}$ by applying Eq. 15 ;

- From Eq. 15, calculate $\mathrm{h}$ as a function of $\mathrm{H}_{\mathrm{R}}$, then apply Eq. 6 to get $\mathrm{h}$;

- Apply Eq. 15 again to get $\mathrm{H}_{\mathrm{R}}$;

- Utilize Eq. 4 to get Ar.

Since $\theta$ and $\mathrm{V}$ are constants, $\mathrm{Ar}$ (accordingly Per) and $\mathrm{H}_{\mathrm{R}}$ will determine the value of $\mathrm{S}$ according to Eq. 7. Also, $\mathrm{H}_{\mathrm{R}}$ is a decreasing function of Ar. The relationship between $\mathrm{H}_{\mathrm{R}}$ or $\mathrm{Ar}$ on the one hand and $\mathrm{S}$ on the other, depends completely on $\omega_{o}$. As can be seen in Figs. 5 and $6, \omega_{o}$ divides the functions $\left(\mathrm{H}_{\mathrm{R}}-\mathrm{S}\right.$ or $\mathrm{Ar}-\mathrm{S}$ ) into two separate zones:

- Zone (a): where $\omega<\omega_{o}$. In this zone, $\mathrm{S}$ is a decreasing function of $\mathrm{H}_{\mathrm{R}}$ (see Fig. 5) and an increasing function of Ar (see Fig. 6), note that the location of the zones is reversed in this last figure. This means that any increase in room height will decrease its total surface area.

- Zone (b): where $\omega>\omega_{o}$. In this zone, $\mathrm{S}$ is an increasing function of $\mathrm{H}_{\mathrm{R}}$ and a decreasing function of Ar (see Figs. 5 and 6). This means that an increase in $H_{R}$ will increase $\mathrm{S}$. This is the opposite of what happens in zone (a).

Table 1 shows a solved example that sheds more light on the effect of constant $\theta$ and variable Ar on S (Remark 2, case 1). A room has an isosceles triangular shape $\left(\theta=45^{\circ}\right)$ and a volume of $4,000 \mathrm{~m}^{3}$. By applying Eqs. 1 (for $\mathrm{h}$ ), 3 (for Per), 4 (for Ar), 7 (for $S$ ) and 15 (for $\omega_{o}$ ), Table 1 can be calculated. For $\theta=45^{\circ}, \omega_{o}=0.83$ (see Fig. 4). $\mathrm{H}_{\mathrm{R}}$ was assumed for each case except for $\omega_{o}$ (the bold text in the Table). As can be concluded from this table, although increasing $H_{R}$ in the zone 


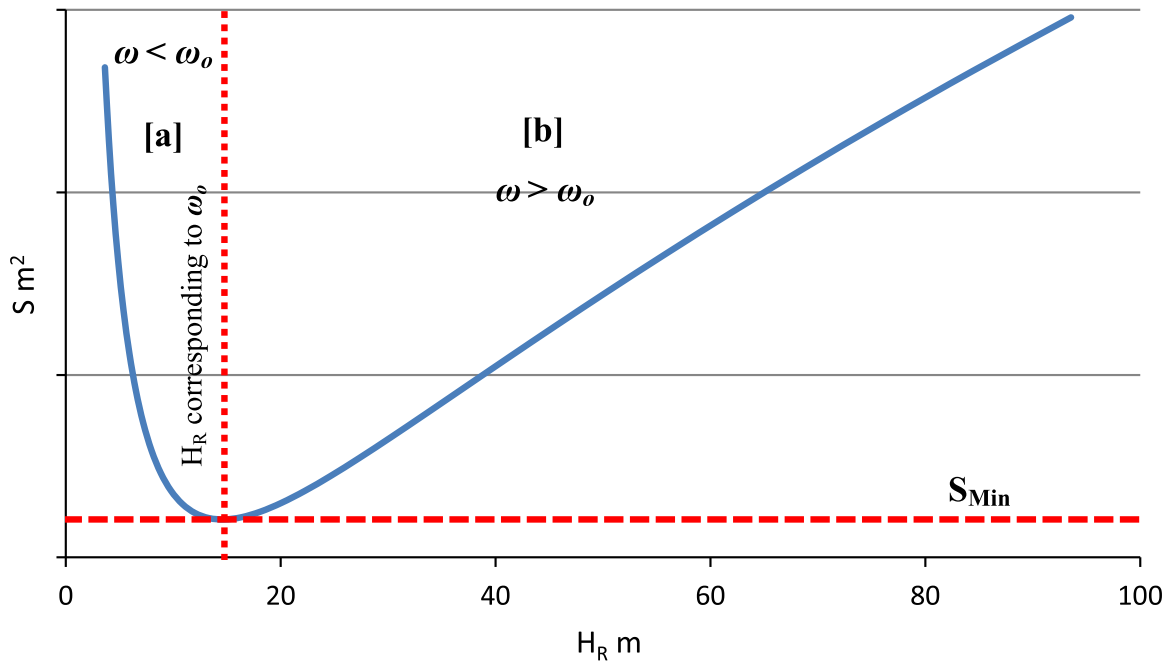

Fig. 5 The relationship of $H_{R}$ to $S$ (case of $\theta=45^{\circ}$ )

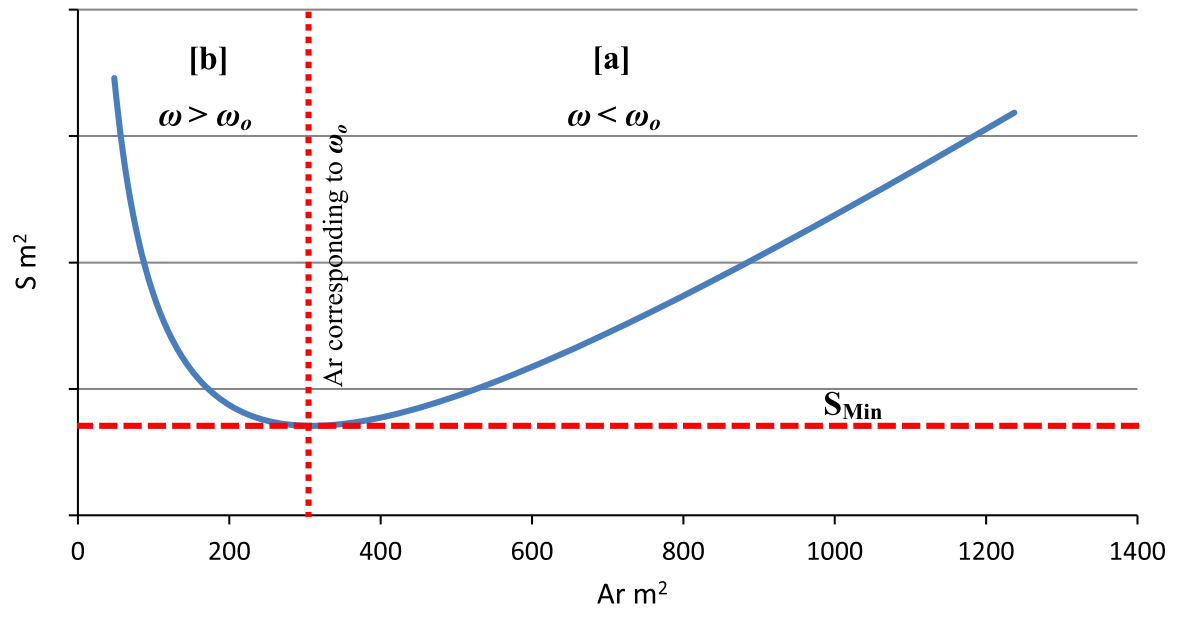

Fig. 6 The relationship of Ar to $S$ (case of $\theta=45^{\circ}$ )

$\omega>\omega_{o}$ increases $\mathrm{S}$, this increase is limited (around 1-3.5\% of S corresponding to $\left.\omega_{o}\right)$. On the contrary, increasing Ar in the zone where $\omega<\omega_{o}$ has a significant effect on S (around 9-52\% of S corresponding to $\omega_{o}$ ).

Case II, Variable $\theta$, Constant $\mathrm{Ar}$ and $\mathrm{H}_{\mathrm{R}}$

This case is perhaps easier than case I. As V, Ar and consequently $\mathrm{H}_{\mathrm{R}}$ are constants for all rooms, thus and according to Eq. 7, the perimeter Per will be the main governor for $\mathrm{S}$. In this case, among the different isosceles triangular rooms with $0^{\circ}<\theta<90^{\circ}, \mathrm{S}_{\text {Min }}$ will occur when the perimeter of the room is minimum. This can 
Table 1 Example shows the effect of $\mathrm{Ar}$ and $\mathrm{H}_{\mathrm{R}}$ on $\mathrm{S}$ (constant $\theta$ and variable $\mathrm{Ar}$ )

\begin{tabular}{|c|c|c|c|c|c|c|c|c|c|}
\hline$\theta^{\circ}$ & $\mathrm{V}\left(\mathrm{m}^{3}\right)$ & $\omega$ (ratio) & & $\mathrm{h}(\mathrm{m})$ & $\mathrm{H}_{\mathrm{R}}(\mathrm{m})$ & $\operatorname{Ar}\left(m^{2}\right)$ & Per $(m)$ & $\mathrm{S}\left(\mathrm{m}^{2}\right)$ & $\Delta \mathrm{S}(\%)$ \\
\hline \multirow[t]{17}{*}{$45^{\circ}$} & \multirow[t]{17}{*}{4,000} & \multirow[t]{8}{*}{$\omega>\omega_{o}$} & 1.47 & 13.97 & 20.50 & 195.12 & 67.45 & $1,772.90$ & 3.43 \\
\hline & & & 1.41 & 14.14 & 20.00 & 200.00 & 68.28 & $1,765.69$ & 3.01 \\
\hline & & & 1.36 & 14.32 & 19.50 & 205.13 & 69.15 & $1,758.76$ & 2.61 \\
\hline & & & 1.31 & 14.51 & 19.00 & 210.53 & 70.06 & $1,752.16$ & 2.22 \\
\hline & & & 1.26 & 14.70 & 18.50 & 216.22 & 71.00 & $1,745.91$ & 1.86 \\
\hline & & & 1.21 & 14.91 & 18.00 & 222.22 & 71.98 & $1,740.05$ & 1.52 \\
\hline & & & 1.16 & 15.12 & 17.50 & 228.57 & 73.00 & $1,734.62$ & 1.20 \\
\hline & & & 1.11 & 15.34 & 17.00 & 235.29 & 74.06 & $1,729.69$ & 0.91 \\
\hline & & $\omega_{o}$ & 0.83 & 16.90 & 14.00 & 285.67 & 81.61 & $1,714.04$ & 0.00 \\
\hline & & \multirow[t]{8}{*}{$\omega<\omega_{o}$} & 0.36 & 22.36 & 8.00 & 500.00 & 107.97 & $1,863.74$ & 8.73 \\
\hline & & & 0.32 & 23.09 & 7.50 & 533.33 & 111.51 & $1,902.97$ & 11.02 \\
\hline & & & 0.29 & 23.90 & 7.00 & 571.43 & 115.42 & $1,950.81$ & 13.81 \\
\hline & & & 0.26 & 24.81 & 6.50 & 615.38 & 119.78 & $2,009.33$ & 17.23 \\
\hline & & & 0.23 & 25.82 & 6.00 & 666.67 & 124.67 & $2,081.35$ & 21.43 \\
\hline & & & 0.18 & 28.28 & 5.00 & 800.00 & 136.57 & $2,282.84$ & 33.18 \\
\hline & & & 0.15 & 29.81 & 4.50 & 888.89 & 143.96 & $2,425.58$ & 41.51 \\
\hline & & & 0.13 & 31.62 & 4.00 & $1,000.00$ & 152.69 & $2,610.75$ & 52.32 \\
\hline
\end{tabular}

Bold values express a special case when $\mathrm{S}$ becomes minimum

be mathematically calculated using Eq. 7 together with Eqs. 1 and 3 when the first derivative of Eq. 3 equals zero, i.e.:

$$
\begin{aligned}
\frac{d s}{d \theta}=\frac{d p e r}{d \theta}= & 2 \sqrt{\frac{A r \sin \theta}{\cos \theta}} \times\left(\frac{-\sin ^{2} \theta-\cos \theta(1+\cos \theta)}{\sin ^{2} \theta}\right)+\left(\frac{1+\cos \theta}{\sin \theta}\right) \\
& \times \sqrt{\frac{\cos \theta}{A r \sin \theta}} \times\left(\frac{A r \cos ^{2} \theta+A r \sin ^{2} \theta}{\cos ^{2} \theta}\right)=0
\end{aligned}
$$

By applying the rules of trigonometry and algebra, Eq. 16 gives:

$$
\cos \theta=0.5 \text {, i.e., } \theta=60^{\circ}
$$

This result comes in a complete agreement with the findings of Remark 1 (see Fig. 3). It also agrees with the mathematical fact that the isosceles triangle has the minimum perimeter among the other triangles (Alsina and Nelsen 2009). Further, the equilateral triangle $\left(\theta=60^{\circ}\right)$ possesses the absolute minimum perimeter, consequently the minimum total surface area among the other rooms that have the same $\mathrm{Ar}$ and $\mathrm{V}$ but different $\theta$.

\section{Remark 3: Walls Ratio $\mathbf{R}_{\mathrm{W}}$}

Walls ratio $R_{W}$ is the ratio between walls surface area $S_{W}$ and room total surface area $\mathrm{S}$ (i.e., $\mathrm{R}_{\mathrm{W}}=\mathrm{S}_{\mathrm{W}} / \mathrm{S}$ ). In our case, this ratio can be mathematically calculated applying the formula: 


$$
R_{W}=\frac{\operatorname{Per} \times H_{R}}{2 A r+\operatorname{Per} \times H_{R}} .
$$

By substitution for Per and Ar from Eqs. 3 and 4, Eq. 18 can be rewritten as:

$$
R_{W}=\frac{H_{R}(1+\cos \theta)}{h \cos \theta+H_{R}(1+\cos \theta)} .
$$

The relationship between $\mathrm{R}_{\mathrm{W}}$ and $\theta$ resembles the relationship between $\mathrm{S}$ and $\theta$ (see Fig. 3), thus $\mathrm{R}_{\mathrm{W}}$ reaches its minimum value when $\theta=60^{\circ}$. In the zone where $\theta<60^{\circ}, \mathrm{R}_{\mathrm{W}}$ is a decreasing function of $\theta$. In the zone where $\theta>60^{\circ}, \mathrm{R}_{\mathrm{W}}$ is an increasing function of $\theta$.

Under the conditions assumed for this work, for a given $\theta$ and according to Eqs. 18 and 19, it is clear that $\mathrm{R}_{\mathrm{W}}$ is a decreasing function of $\mathrm{Ar}$ (or $\mathrm{h}$ ) and increasing function of $\mathrm{H}_{\mathrm{R}}$. This is a logical conclusion as long as $\mathrm{V}$ is constant. What should be mentioned in this context is the special case of the isosceles triangular right prism when its dimensions fulfill $\omega_{o}$, this special $\mathrm{R}_{\mathrm{W}}$ will be called the critical walls ratio $R_{W o}$. To calculate $R_{W o}$, the conditions for $\omega_{o}$ must be applied, thus, Eq. 19 can be rewritten as:

$$
R_{W o}=\frac{\frac{2 h(1+\cos \theta)}{(\sec \theta+1)}}{h \cos \theta+\frac{2 h(1+\cos \theta)}{(\sec \theta+1)}} .
$$

This leads to:

$$
R_{W o}=\frac{2}{3} .
$$

This means that $R_{W o}$ is constant for any $\theta\left(0^{\circ}<\theta<90^{\circ}\right)$ and is equal to $2 / 3$.

\section{Remark 4: Case of Numerical Equality}

For the room under discussion, two cases of numerical equality will be examined. The first considers the numerical equality between the perimeter Per and the floor area Ar. The last considers the numerical equality between the total surface area $S$ and the volume $\mathrm{V}$.

Case I: Equality of Per and Ar

In this case and according to Eqs. 3 and 4, the numerical equality between Per and Ar occurs when:

$$
2 h\left(\frac{1+\cos \theta}{\sin \theta}\right)=\frac{h^{2}}{\tan \theta} .
$$

The value of $\mathrm{h}$ that fulfills the equality will be called the critical altitude $h_{o}$. By applying the rules of algebra and trigonometry, Eq. 22 can be rewritten as:

$$
h_{o}=2(\sec \theta+1) \text {. }
$$




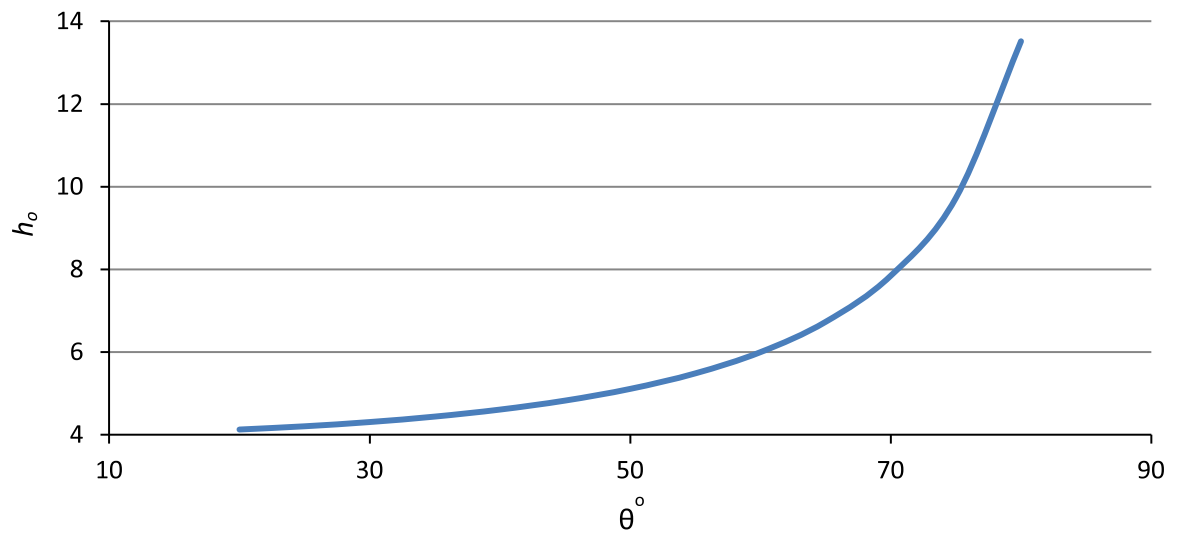

Fig. 7 Values of $h_{o}$ in the range $20^{\circ} \leq \theta \leq 80^{\circ}$ according to Eq. 23

Equation 23 reveals the condition under which both Per and Ar will be equal. Like $\omega_{o}$, it is clear from Eq. 23 that this case of numerical equality depends entirely on $\theta$, and for every $\theta$ there is a specific $h_{o}$ for which Per equals Ar. The values of $h_{o}$ in the range $20^{\circ} \leq \theta \leq 80^{\circ}$ are presented in Fig 7. It is clear from this figure that $h_{o}$ is an increasing function of $\theta$. The values of $b$, Per and Ar can be calculated from Eqs. 2, 3 and 4.

\section{Case II: Equality of S and V}

In this case, the numerical equality between $\mathrm{S}$ and $\mathrm{V}$ occurs when:

$$
2 A r+\left(\operatorname{Per} \times H_{R o}\right)=A r \times H_{R o}
$$

where $H_{R o}$ is the critical room height that fulfills this equality. Based on Eqs. 3 and 4, and applying the rules of algebra and trigonometry, Eq. 24 can be rewritten as:

$$
H_{R o}=\frac{2 h}{h-2(\sec \theta+1)} .
$$

Thus, according to Eq. 25, for every $\theta$ and Ar, there is a specific $H_{R o}$ such that $\mathrm{S}$ and $\mathrm{V}$ are numerically equal. This can be calculated in the following sequence:

- Determine both $\theta$ and Ar for the room;

- Apply Eq. 1 to get h;

- Substitute in Eq. 25 to get the critical room height $H_{R o}$, the height of the prism that fulfills the numerical equality between $\mathrm{S}$ and $\mathrm{V}$.

The minus sign (-) in the denominator of Eq. 25 also reveals that there are acceptable and unacceptable range in this equation. In other words, for every $\theta$ there is a minimum $\mathrm{h}$ (accordingly $\mathrm{Ar}$ ) under which the numerical equality between $\mathrm{S}$ and $\mathrm{V}$ will never exist. This occurs when $H_{R o}$ tends to $\infty$, i.e., $\mathrm{h}=2(\sec \theta+1)$, or $\mathrm{Ar}$ equals Per according to Eq. 23. Figure 8 represents the relationship between Ar and $H_{R o}$ calculated from Eq. 25 (for $\theta=45^{\circ}$ ). As can be seen from the figure, in the 


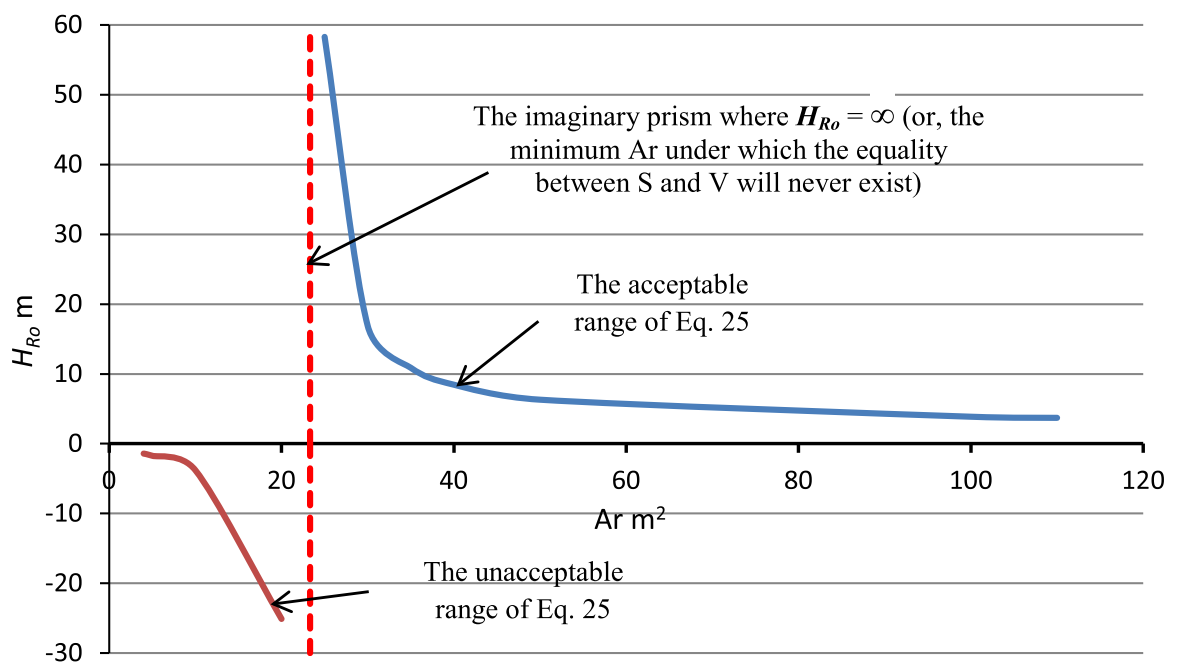

Fig. 8 The relationship of $\mathrm{Ar}$ and $H_{R o}$ (case of $\theta=45^{\circ}$ )

acceptable range, $H_{R o}$ is a decreasing function of Ar while the function can be divided into two main zones, zone of rapid decay (when Ar tends to be equal to Per) and zone of slow decay (when Ar is far from this equality).

\section{Conclusions}

This work has examined the interrelationships between the dependent and independent variables that control the values of the measures Per, Ar, S and V and how these variables affect each other in the case of regular triangular right prisms. Under the conditions assumed for this work, four remarks were concluded. In the first, the effect of $\theta$ on $\mathrm{S}$ was investigated. In the second remark, the minimum total surface area $S_{\text {Min }}$ for the room under discussion was calculated in two cases, case of constant $\theta$ and case of variable $\theta$. In the first case, new variable $\left(\omega=\mathrm{H}_{\mathrm{R}} / \mathrm{h}\right)$ was introduced. For every $\theta$ there is a specific $\omega$ (called $\omega_{o}$ ) that results $\mathrm{S}_{\mathrm{Min}}$. Results showed that $\omega_{o}$ depends entirely on $\theta$. The values of $\omega_{o}$ in the range $20^{\circ} \leq \theta \leq 80^{\circ}$ were calculated and presented. In the second case, where $\theta$ is variable, results showed that $\mathrm{S}_{\text {Min }}$ corresponds to $\theta=60^{\circ}$. The third remark calculates walls ratio $R_{W}$, results showed that $R_{W}$ reaches its minimum value when $\theta=60^{\circ}$. In case of the isosceles triangular right prism that has dimensions fulfill $\omega_{o}, \mathrm{R}_{\mathrm{W}}$ was called $R_{W o}$. Results showed that $R_{W o}$ is constant (=2/3) regardless the value of $\theta$.

The last remark investigates the conditions for the numerical equality either between Per and Ar or S and V. In the first case, another variable $\left(h_{o}\right)$ was introduced. Results showed that $h_{o}$ depends also on $\theta$. For every $\theta$ there is a specific $h_{o}$ that fulfills the numerical equality between Per and Ar. The values of $h_{o}$ in the range $20^{\circ} \leq \theta \leq 80^{\circ}$ were calculated and presented. In the second case, the 
condition for the numerical equality between $\mathrm{S}$ and $\mathrm{V}$ was calculated. Results showed that for every $\theta$ and Ar, there is a specific $\mathrm{H}_{\mathrm{R}}$ (called $H_{R o}$ ) that fulfills this equality. Results also showed that for every $\theta$ there is a minimum $\mathrm{h}$ under which this equality will never exist. This corresponds to $h_{o}$ (i.e., $\mathrm{Ar}=\mathrm{Per}$ ).

Acknowledgments The author would like to express his gratitude and sincere appreciation to all colleagues who make this work possible. In particular, thanks to Prof. Dr. Morad Abdel Kader, Dr. Esraa Elkhateeb and Dr. Ahmed Zakareia for their valuable discussion and help during the analysis.

\section{References}

Alsina, Claudi, and Nelsen, Roger. 2009. When less is more? visualizing basic inequalities. Mathematical association of America

Bird, John. 2003. Engineering Mathematics. 4th edn. Newnes

Ching, Francis, D.K. 2007. Architecture: form, space, and order. 3rd edn. Wiley.

Elkhateeb, Ahmed. 2012. Form-acoustics relationship, effect of room shape and volume on the quality of speech. Lambert Academic Publishing

Ferguson, D.F., and Piggott, H.E. 1923. Areas and volumes: their accurate and approximate determination. Constable \& company ltd

Gieck, Kurt, and Gieck, Reiner. 2006. Engineering formulas. 8th edn. McGraw-Hill

Grondzik, Walter, T., Kwok, Alison, Stein, Benjamin, and Reynolds, John. 2006. Mechanical and electrical equipment for buildings. 10th edn. Wiley.

Konya, Allan. 2011." Cairo: Anglo-Egptian Bookshop. (Translated by Ahmed Elkhateeb) (Arabic trans. of Konya, Allan, Design Primer for Hot Climates, 2nd ed., London, Architectural Press, 1984).

Sabine, Wallace. 1993. Collected papers on acoustics. California: Peninsula publishing

Ahmed Elkhateeb is an Egyptian architect who graduated in 1990 from the Department of Architecture, Faculty of Engineering of Ain Shams University (Cairo, Egypt). He worked in the same department until summer 2011. He completed his M.Sc. and Ph.D. in the field of architectural acoustics, which is his primary area of interest along with mathematics and its relation with architecture. He has many published researches in national and international scholarly journals in the field of building science, in addition to five published books in the same research area. He also supervised many theses in different areas related to architecture. He was awarded two international research Grants in the field of architectural acoustics. As of fall 2012 he is Full Professor of Architecture and Building Science in the Department of Architecture in the Faculty of Environmental Designs of King Abdulaziz University, Jeddah, Saudi Arabia. 